Original Research article

\title{
Identification of a new subtype of dipeptidyl peptidase 11 and a third group of the S46-family members specifically present in the genus Bacteroides
}

\author{
Takayuki K. Nemoto a, ${ }^{\text {, }}$ Gustavo Arruda Bezerra ${ }^{\mathrm{b}, 1}$, Toshio Ono ${ }^{\mathrm{a}}$, \\ Haruka Nishimata ${ }^{c}$, Taku Fujiwara ${ }^{c}$, and Yuko Ohara-Nemoto ${ }^{a}$ \\ a Department of Oral Molecular Biology, Course of Medical and Dental Sciences, Nagasaki \\ University Graduate School of Biomedical Sciences, Nagasaki 852-8588, Japan \\ ${ }^{\mathrm{b}}$ Department of Structural and Computational Biology, Max F. Perutz Laboratories, University of \\ Vienna, Vienna Biocenter, Vienna Biocenter Campus 5, 1030 Vienna, Austria \\ ${ }^{c}$ Department of Pediatric Dentistry, Course of Medical and Dental Sciences, Nagasaki University \\ Graduate School of Biomedical Sciences, Nagasaki 852-8588, Japan \\ * Corresponding author. Takayuki K. Nemoto, Department of Oral Molecular Biology, Course of \\ Medical and Dental Sciences, Nagasaki University Graduate School of Biomedical Sciences, 1-7-1 \\ Sakamoto, Nagasaki 852-8588, Japan. Tel.: +81 95819 7640; Fax: +81 958197642
}

E-mail address: tnemoto@nagasaki-u.ac.jp (T.K. Nemoto), gustavo.arrudabezerra@sgc.ox.ac.uk (G.A. Bezerra), ono-t@nagasaki-u.ac.jp (T. Ono), nishimata@nagasaki-u.ac.jp (H. Nishimata), takufuji@nagasaki-u.ac.jp (T. Fujiwara), ynemoto@nagasaki-u.ac.jp (Y. Ohara-Nemoto).

${ }^{1}$ Present address: Structural Genomics Consortium, University of Oxford, Oxford OX3 7DQ, UK 


\section{ABSTRACT}

Peptidase family S46 consists of two types of dipeptidyl-peptidases (DPPs), DPP7 and DPP11, which liberate dipeptides from the N-termini of polypeptides along with the penultimate hydrophobic and acidic residues, respectively. Their specificities are primarily defined by a single amino acid residue, Gly ${ }^{673}$ in DPP7 and $\mathrm{Arg}^{673}$ in DPP11 (numbering for Porphyromonas gingivalis DPP11). Bacterial species in the phyla Proteobacteria and Bacteroidetes generally possess one gene for each, while Bacteroides species exceptionally possess three genes, one gene as DPP7 and two genes as DPP11, annotated based on the full-length similarities. In the present study, we aimed to characterize the above-mentioned Bacteroides S46 DPPs. A recombinant protein of the putative DPP11 gene BF9343_2924 from Bacteroides fragilis harboring Gly ${ }^{673}$ exhibited DPP7 activity by hydrolyzing Leu-Leu-4-methylcoumaryl-7-amide (MCA). Another gene, BF9343_2925, as well as the Bacteroides vulgatus gene (BVU_2252) with $\operatorname{Arg}^{673}$ was confirmed to encode DPP11. These results demonstrated that classification of S46 peptidase is enforceable by the S1 essential residues. Bacteroides DPP11 showed a decreased level of activity towards the substrates, especially with P1-position Glu. Findings of 3D structural modeling indicated three potential amino acid substitutions responsible for the reduction, one of which, Asn650Thr substitution, actually recovered the hydrolyzing activity of Leu-Glu-MCA. On the other hand, the gene currently annotated as DPP7 carrying Gly $^{673}$ from B. fragilis (BF9343_0130) and Bacteroides ovatus (Bovatus_03382) did not hydrolyze any of the examined substrates. The existence of a phylogenic branch of these putative Bacteroides DPP7 genes classified by the C-terminal conserved region $\left(\right.$ Ser $^{571}$-Leu $\left.{ }^{700}\right)$ strongly suggests that Bacteroides species expresses a DPP with an unknown property. In conclusion, the genus Bacteroides exceptionally expresses three S46-family members; authentic DPP7, a new subtype of DPP11 with substantially reduced specificity for Glu, and a third group of S46 family members. 


\section{Highlights}

- Classification by the S1 residue $\mathrm{Arg}^{673} / \mathrm{Gly}^{673}$ is reliable for S46 peptidase.

- Three S46 DPPs are exceptionally present in the genus Bacteroides.

- One of Bacteroides DPP with Gly ${ }^{673}$ represented authentic DPP7.

- Another DPP with Gly ${ }^{673}$ is a third group of S46 members with an unknown property.

- The Bacteroides DPP with $\mathrm{Arg}^{673}$ is a new type of DPP11.

Keywords: Bacteroides, DPP7, DPP11, Porphyromonas gingivalis, S46 peptidase 


\section{Introduction}

Dipeptidyl-peptidase (DPP) 7 and DPP11 were first discovered in Porphyromonas gingivalis [1, 2] and Porphyromonas endodontalis [2], Gram-negative asaccharolytic anaerobes implicated as causative agents of chronic [3, 4] and acute [5,6] periodontal diseases, respectively. These periodontal pathogens solely metabolize amino acids as carbon and energy sources, hence, their bulk degradation process of extracellular peptides is important for microorganism survival. In addition, another characteristic feature of Porphyromonas species is incorporation of amino acids mainly as dipeptides, not as single amino acids. These features seem to convey an advantage for Porphyromonas species to form subgingival periodontopathic microbial symbioses with other bacterial species, such as saccharolytic Aggregatibacter actinomycetemcomitans and Prevotella intermedia, and also Fusobacterium and Prevotella species that incorporate single amino acids.

In $P$. gingivalis, extracellular nutritional proteins are initially digested to oligopeptides by two potent types of cysteine endopeptidases, Arg-gingipain [7] and Lys-gingipain (C25.002) [8], then further degraded into di- and tri-peptides by exopeptidases, i.e., DPPs [1, 2, 9-11] and prolyl tripeptidyl-peptidase A (PTP-A) [12, 13], respectively, together with the dipeptide-releasing activity of Arg- and Lys-gingipains [11]. To date, five DPPs have been identified in P. gingivalis, i.e., DPP4, which mainly releases an Xaa-Pro (Xaa, any amino acid) dipeptide and also Xaa-Ala to a lesser extent $[9,10,14,15]$, DPP5, with a preference for Ala and aliphatic residues at the penultimate position from the N-terminus [11], DPP7 [1], and DPP11 [2]. Although the gene encoding DPP3 (generally referred to as DPPIII) specific for Arg (M49.003) is also present, this DPP does not seem to be involved in extracellular protein metabolism, because DPP3 appears to be located in the cytoplasm [11]. Additionally, acylpeptidyl oligopeptidase (AOP) produces di- and tri-peptides preferentially from $\mathrm{N}$-terminally acylated peptides [16]. Therefore, in consideration of the fact that 
most extracellular polypeptides derived from human serum are $\mathrm{N}$-terminally acylated, proteinaceous nutrients in gingival crevicular fluid should be effectively degraded into di- and tri-peptides, and then incorporated into P. gingivalis by co-operative actions of these peptidases [17]. DPP4, DPP5, PTP-A, and AOP belong to the peptidase family S9, and DPP7 and DPP11 are classified into the S46 peptidase.

Currently, the S46 family is composed of three members, DPP7 (S46.001), DPP11 (S46.002), characterized in $P$. gingivalis (PgDPP7, PgDPP11, respectively), and DAPBII from Pseudoxanthomonas mexicana (S46.003) [18, 19]. Since the biochemical property and substrate preference of DAPBII are nearly identical to those of PgDPP7, the S46 family should be composed solely of two members, i.e., DPP7 preferentially liberates dipeptides with both P1 and P2 hydrophobic residues, and DPP11 is specific for P1 Asp and Glu. Interestingly, we previously found that each of their P1 substrate specificities is primarily defined by a single amino acid residue, $\mathrm{Gly}^{673}$ in DPP7 and $\mathrm{Arg}^{673}$ in DPP11 (numbering in PgDPP11) [20]. In Shewanella DPP11, $\operatorname{Arg}^{673}$ is substituted by Ser, then Lys ${ }^{680}$ plays the role of S1. Recently, the 3D structure of DPP11 was reported [21,22], and analysis of the complex of di-, tri-, and tetra-peptides suggested that an increase in conformational entropy of DPP11 is the primary driving force for substrate recognition [22].

Orthologs of DPP7 and DPP11 are widely distributed in the phyla Proteobacteria and Bacteroidetes, and these bacteria generally possess one each of the DPP7 and DPP11 genes. However, it was unexpectedly observed that the genus Bacteroides contain three members of the S46 family, one is DPP7 while the other two are DPP11 genes, annotated based on full-length similarity. In contrast, our previous proposal regarding classification by $\mathrm{Gly}^{673}$ and $\mathrm{Arg}^{673}$ indicates the existence of two DPP7 and one DPP11 gene in Bacteroides species. Accordingly, biochemical and enzymatic analyses are indispensable to determine these entities. 
In the present study, recombinant forms of putative Bacteroides DPP7 and DPP11 genes were expressed exogenously, and their substrate specificities were determined for classification. Our results clearly show the validity of essential $\mathrm{Gly}^{673}$ and $\mathrm{Arg}^{673}$ residues, and the inappropriateness of full-length similarities for classification of the family of S46 DPPs. Furthermore, we found that Bacteroides DPP11 specifically exhibits decreased activity compared to authentic DPP11, especially for substrates with P1-position Glu. Also, 3D modeling and substitution analysis

demonstrated that $\mathrm{Asn}^{650}$ is partially responsible for this phenomenon. Additionally, the present findings indicate the existence of the third S46 member, though its real role remains unknown.

\section{Materials and methods}

\subsection{Materials}

pQE60 (Qiagen Inc., Chatsworth, CA) and pTrcHis2-TOPO (Invitrogen, Carlsbad, CA) were used as expression vectors. Restriction and DNA-modifying enzymes were purchased from Takara Bio (Tokyo, Japan) and New England Biolabs (Ipswich, MA), respectively. Quick Taq HS DyeMix and KOD-Plus-Neo DNA polymerase came from Toyobo (Tokyo, Japan). Met-Leu- and Leu-Asp-MCA were obtained from the Peptide Institute (Osaka, Japan). Leu-Glu-, Leu-Leu-, Phe-Leu-, and His-Leu-MCA were synthesized by Thermo Fisher Scientific (Ulm, Germany) and Scrum (Tokyo, Japan). Oligonucleotide primers were synthesized by FASMAC (Atsugi, Japan). Low-molecular-weight marker was obtained from GE Healthcare (Little Chalfont, UK). Genomic DNA from B. fragilis strain NCTC 9343 (JCM 11019 ), Bacteroides vulgatus JCM 5826 ${ }^{\mathrm{T}}$, Bacteroides thetaiotaomicron JCM $5827^{\mathrm{T}}$, Bacteroides ovatus RDB 09006, and Bacteroides dorei 
JCM 13471 were provided by the RIKEN BRC through the National Bio-Resource Project of the MEXT, Japan. Bacterial strains B. fragilis YCH10, B. thetaiotaomicron KYU12, and Bacteroides uniformis KYU12 were generous gifts from Dr. Mariko Naito of Nagasaki University.

\subsection{Culture Conditions}

P. gingivalis strain ATCC 33277, B. fragilis YCH10, B. thetaiotaomicron KYU12, B. uniformis KYU12, and $B$. dorei were grown anaerobically $\left(80 \% \mathrm{~N}_{2}, 10 \% \mathrm{CO}_{2}, 10 \% \mathrm{H}_{2}\right)$ in Anaerobic Bacterial Culture Medium broth (Eiken Chemical, Tokyo, Japan), supplemented with 0.5 $\mu \mathrm{g} / \mathrm{ml}$ of menadione. Following centrifugation, bacterial cells were suspended in phosphate-buffered saline (PBS) at $\mathrm{pH} 7.4$, then centrifuged at $6000 \times \mathrm{g}$ for $15 \mathrm{~min}$ at $4{ }^{\circ} \mathrm{C}$. Cell pellets were re-suspended in PBS to adjust absorbance at $600 \mathrm{~nm}$ to 5.0, then used for DPP assays.

\subsection{Construction of Expression Plasmids}

The DNA fragment of BVU_2252, deduced as B. vulgatus S46 peptidase (Asp ${ }^{20}-\mathrm{Glu}^{728}$ ) (UniProtKB, YP_001299533.1/MEROPS code MER034614), was amplified by PCR using a set of primers (Table 1), with genomic DNA used as a template, then the PCR product was ligated with pTrcHis2-TOPO according to the manufacturer's protocol. DNA fragments of the $B$. ovatus genes, Bovatus_00118 $\left(\mathrm{Asn}^{2}-\mathrm{Glu}^{719}\right)$ (MEROPS code, MER109242) and Bovatus_03382 (Lys ${ }^{2}-\mathrm{Arg}^{713}$ ) (MER09141), were amplified by PCR using sets of primers. The former and latter fragments were digested by BamHI and BglII, respectively, then inserted into the BamHI site of pQE60. DNA fragments of the B. fragilis genes, BF9343_0130 (Asn ${ }^{2}-\mathrm{Gly}^{725}$, MEROPS code MER039992), and BF9343_2925 (Met ${ }^{2}-$ Glu $^{721}$, MER039991), were amplified by PCR using sets of primers. The PCR 
fragments were digested by BamHI, then inserted into the BamHI site of pQE60. Expression plasmids of P. gingivalis DPP7 and DPP11, B. fragilis DPP7 (BF9343_2924), P. endodontalis, B. vulgatus, Capnocytophaga gingivalis, Flavobacterium psychrophilum, and Shewanella putrefaciens DPP11 have been reported [2, 11, 20, 23].

\subsection{Amino Acid Numbering of DPP7 and DPP11}

The amino acid numbers of all recombinant proteins were counted as those of PgDPP11 to avoid confusion. Accordingly, since Gly ${ }^{666}$ of PgDPP7, Gly ${ }^{678}$ of BF9343_0130, Gly ${ }^{674}$ of BF9343_2924, $\operatorname{Arg}^{674}$ of BF9343_2925, and $\operatorname{Arg}^{670}$ of P. endodontalis DPP11 (PeDPP11) are equivalent to $\operatorname{Arg}^{673}$ of PgDPP11, they were numbered as 673. In addition, Asn ${ }^{651}$, Gly ${ }^{671}$, and $\mathrm{Leu}^{679}$ of BfDPP11 are equivalent to PgDPP11 $\mathrm{Thr}^{650} \mathrm{Asn}^{670}$, and $\mathrm{Val}^{678}$, respectively, therefore their numbers were unified to 650,670 , and 678 , respectively.

\subsection{In vitro Mutagenesis}

PCR-based in vitro mutagenesis was carried out to substitute Gly ${ }^{673}$ to Arg in the expression plasmids of B. fragilis BF9343_0130 and BF9343_2924 using primer sets (Table 1), as previously reported [2, 20]. Substitutions of Asn650Thr, Gly670Asn, and Leu678Val in BfDPP11 (BF9343_2925) were performed, and Thr650Asn and Asn670Gly in the plasmid for PgDPP11 were inversely produced using sets of primers. Additionally, the double mutations of Asn650Thr and Leu678Val were introduced into the plasmid for BfDPP11.

\subsection{Expression and Purification of Recombinant Proteins}


Escherichia coli XL-1 Blue cells carrying respective expression plasmids were cultured in Luria-Bertani broth supplemented with $75 \mu \mathrm{g} / \mathrm{ml}$ of ampicillin at $37^{\circ} \mathrm{C}$. Recombinant proteins were induced with $0.2 \mathrm{mM}$ isopropyl- $\beta$-thiogalactopyranoside at $30{ }^{\circ} \mathrm{C}$ for $4 \mathrm{~h}$, then purified from cell lysates using Talon affinity chromatography, as previously reported [2]. Purified samples were stored at $-80{ }^{\circ} \mathrm{C}$ until use.

\subsection{Measurement of DPP Activity}

DPP7 activity was measured with Phe-Leu- and Leu-Leu-MCA, and DPP11 activity was determined using Leu-Asp- and Leu-Glu-MCA, as previously reported [11]. Briefly, the reaction was started by addition of recombinant proteins $(4-200 \mathrm{ng})$ or bacterial cell suspensions ( $4 \mu \mathrm{l}$ of $\left.\mathrm{A}_{600}=5\right)$ in a reaction mixture $(200 \mu \mathrm{l})$ composed of $50 \mathrm{mM}$ sodium phosphate $(\mathrm{pH} 7.0), 5 \mathrm{mM}$ EDTA, and $20 \mu \mathrm{M}$ dipeptidyl MCA. After $30 \mathrm{~min}$ at $37{ }^{\circ} \mathrm{C}$, fluorescence intensity was measured with excitation at $380 \mathrm{~nm}$ and emission at $460 \mathrm{~nm}$. Activity is expressed as pmol $\mathrm{min}^{-1} \mu^{-1}$ of protein.

To determine enzymatic parameters, recombinant proteins were incubated with various concentrations of dipeptidyl MCA. Data were analyzed using a nonlinear regression curve fitted to the Michaelis-Menten equation with the GraphPad Prism software program (San Diego, CA). Values were calculated from three independent measurements and are shown as average \pm S.D.

\subsection{D Modeling of BfDPP11}

In order to obtain insight regarding the 3-dimensional structure of BfDPP11 and particularly 
its active site, we employed homology modeling. The model was generated via the server Phyre2 (Protein Homology/Analogy Recognition Engine) [24] using the one-to-one threading tool in expert mode. Similar to PeDPP11, BfDPP11 is expected to undergo a conformational change upon peptide binding, resulting in complete formation of the active site. Therefore, PeDPP11 in complex with a peptide was chosen as a template for the modeling routine. The coordinates of PeDPP11 in complex with the dipeptide Arg-Asp (PDB code: 5JWG) [22] were subjected to structural alignment and further model building.

\subsection{Construction of Phylogenic Trees}

In the MEROPS database (release 9.6), 264 members of the S46 family were chosen for construction of phylogenic trees, with ClustalX software [25] used to align the sequences. The conserved amino acid sequence at the C-terminal region $\left(\operatorname{Pro}^{571}-\mathrm{Leu}^{700}\right.$ of PgDPP11) was used for generating the phylogenic trees. Phylogenic analysis with the neighbor-joining (NJ) algorithm [26] was conducted using MEGA version 5 [27].

\subsection{Miscellaneous}

Recombinant proteins $(0.4 \mu \mathrm{g})$ were separated by PAGE in the presence of $0.1 \%(\mathrm{w} / \mathrm{v})$ of SDS with a polyacrylamide concentration of $10 \%(\mathrm{w} / \mathrm{v})$. Separated proteins were stained with Coomassie Brilliant Blue. Protein concentrations were determined by the Coomassie Brilliant Blue dye method (Bio-Rad) using bovine albumin as a standard. 


\section{Results}

\subsection{Characterization of Three S46-Family Members in the Genus Bacteroides}

To date, commercially available synthetic substrates, Ala-Phe-p-nitroanilide [1], and Lys-Alaand Met-Leu-MCA [28], had been used for measuring DPP7 activity. However, since we established that DPP7 prefers hydrophobic residues at the P2 as well as P1 position [23], we initially synthesized Leu-Leu- and Phe-Leu-MCA with hydrophobic residues at both positions to test the potentials as DPP7 substrates. The $k_{\text {cat }} / K_{\mathrm{m}}$ values of PgDPP7 were $37.7 \mu \mathrm{M}^{-1} \mathrm{sec}^{-1}$ for Leu-Leu-MCA and $15.3 \mu \mathrm{M}^{-1} \mathrm{sec}^{-1}$ for Phe-Leu-MCA, which were much higher than those for Met-Leu-, His-Ala- (Table 2), and Lys-Ala-MCA (data not shown). Thus, Leu-Leu- and Phe-Leu-MCA were used in the present study for measurement of DPP7 activity.

Species of the genus Bacteroides frequently contain three genes of the S46 family (Supplemental Table S1), and their annotation based on the full-length similarity to PgDPP7 and PgDPP11 was confusing. For example, two genes (BF9343_2924, BF9343_2925) in B. fragilis are annotated as DPP11 (Table 3); BF9343_2925 with $\operatorname{Arg}^{673}$ seemed to be DPP11, while BF9343_2924 carrying Gly ${ }^{673}$ could be DPP7 according to the classification of DPP7 with the S1 Gly ${ }^{673}$ residue. Similarly, the B. ovatus putative DPP11 gene (Bovatus_00118) with Gly ${ }^{673}$ was considered to be DPP7. To determine the enzymatic characteristics, three S46-family members of B. fragilis and two from B. ovatus were expressed in E. coli, and purified to homogeneity (Fig. 1). One of the putative DPP11 genes, BF9343_2925 (MER039991) ( $\left.\operatorname{Arg}^{673}\right)$, expressed a protein truly hydrolyzing Leu-Asp-MCA efficiently and Leu-Glu-MCA weakly, confirming its DPP11 entity (BfDPP11). Meanwhile, another DPP11 gene, BF9343_2924 (MER039993) with Gly ${ }^{673}$, expressed a protein specifically hydrolyzing Leu-Leu-MCA and not Leu-Asp/Glu-MCA, exhibiting its entity as DPP7 
(BfDPP7). These findings demonstrated that the presence of Gly ${ }^{673}$ is a definitive feature of DPP7, while the annotation based on the full-length amino acid sequence similarity misled the identification.

In addition, the enzymatic property of the putative DPP7 gene BF9343_0130 (MER039992) $\left(\mathrm{Gly}^{673}\right)$ was examined. BF9343_0130, which shows approximately 43\% amino acid similarity to PgDPP7, was successfully expressed and purified (Fig. 1). However, the purified protein hydrolyzed neither Leu-Leu- nor Leu-Asp/Glu-MCA, and did not hydrolyze any other synthetic substrates for DPPs, such as Gly-Pro-, Ser-Tyr-, Gly-Phe-, Met-Leu-, Lys-Leu-, and Lys-Val-MCA (data not shown). Since we previously demonstrated that PgDPP7 bearing the mutation Gly673Arg partially acquired a DPP11-like property [20], the Gly673Arg substitution was introduced into BF9343_0130 as well as BF9343_2924/BfDPP7. As a result, BF9343_2924/BfDPP7 Gly673Arg slowly hydrolyzed Leu-Glu-MCA, showing a change into the DPP11 type. However, BF9343_0130 Gly673Arg was still not able to hydrolyze any substrates. These results further suggested a distant relationship of BF9343_0130 $\left(\right.$ Gly $\left.^{673}\right)$ with BF9343_2924/BfDPP7 (Gly $\left.{ }^{673}\right)$ as well as BF9343_2925/BfDPP11 ( $\left.\operatorname{Arg}^{673}\right)$.

As another DPP7 gene in Bacteroides species carrying Gly ${ }^{673}$, we examined Bovatus_00118, annotated as DPP11, and Bovatus_03382, annotated as DPP7, in B. ovatus. As shown in Fig. 1, Bovatus_00118 $\left(\mathrm{Gly}^{673}\right)$ was not correctly annotated, but was truly DPP7 (BvDPP7) and selectively hydrolyzed Leu-Leu-MCA. In addition, similar to B. fragilis BF9343_0130 (Gly ${ }^{673}$ ), putative $B$. ovatus DPP7 (Bovatus_03382, Gly ${ }^{673}$ ) did not hydrolyze any synthetic MCA substrates. The sequence similarity between Bovatus_03382 and BF9343_0130 was close to 77\%, and that between BF9343_2924/BfDPP7 and Bovatus_00118/BvDPP7 was around 84\%, while the similarities between all other combinations of two proteins within the species were lower (Table 3). Therefore, BF9343_0130 from B. fragilis and Bovatus_03382 from B. ovatus are not DPP7, and considered 
classifiable as novel DPP. Taken together, our findings revealed that gene annotation based on full-length amino acid similarities did not match biochemical identification of the S46 peptidases DPP7 and DPP11. In addition, the Bacteroides S46 DPP genes carrying Gly ${ }^{673}$, which are currently classified as DPP7, should be considered as a third group of S46 family members.

\subsection{Hydrolyzing Properties of DPP11 from Genus Bacteroides}

We previously reported the presence of two types of DPP11 in respect to substrate specificity; DPP11 with $\operatorname{Arg}^{673}$ from P. gingivalis, P. endodontalis, C. gingivalis, and F. psychrophilum, with higher $k_{\text {cat }} / K_{\mathrm{m}}$ values for Leu-Asp-MCA, and DPP11 with $\mathrm{Ser}^{673} / \mathrm{Lys}{ }^{680}$ from S. putrefaciens with a higher $k_{\text {cat }} / K_{\mathrm{m}}$ value for Leu-Glu-MCA [20]. In order to comprehensively understand the Asp/Glu preference in DPP11, we reexamined the enzymatic characteristics of DPP11s from $P$. gingivalis, $P$. endodontalis, C. gingivalis, F. psychrophilum, S. putrefaciens B. fragilis, and B. vulgatus (Fig. 2). Purified recombinant DPP11s were found to migrate at 70 - to $85-\mathrm{kDa}$ positions on SDS-PAGE, reflecting their calculated molecular masses. All DPP11 molecules except for S. putrefaciens DPP11 showed hydrolyzing activities more preferential for Leu-Asp- to Leu-Glu-MCA, though B. vulgatus DPP11 (BvDPP11) displayed extremely low activities. The ratios of activity toward Leu-Glu-MCA per those for Leu-Asp-MCA of $P$. gingivalis, $P$. endodontalis, and C. gingivalis DPP11 were 0.47 , 0.34 , and 0.34 , respectively, while the ratios of BfDPP11 and BvDPP11 were substantially lower (0.014 and 0.018 , respectively). The $k_{\text {cat }} / K_{\mathrm{m}}$ value of BfDPP11 for Leu-Asp-MCA was $40.9 \pm 4.4$ $\left(\mu \mathrm{M}^{-1} \sec ^{-1}\right)$ and for Leu-Glu-MCA was $0.87 \pm 0.04\left(\mu \mathrm{M}^{-1} \sec ^{-1}\right)$, indicating that BfDPP11 cleaves Leu-Asp-MCA at a level 47-fold more efficiently as compared to Leu-Glu-MCA. Hence, even though the substantially low $k_{\text {cat }} / K_{\mathrm{m}}$ value of BfDPP11 for Leu-Glu-MCA was caused by both a decrease in $k_{\text {cat }}$ and an increase in $K_{\mathrm{m}}$, it was evident that the increase in $K_{\mathrm{m}}$ had a larger impact on 
that change (Table 2).

Next, we investigated DPP11 activity in Bacteroides cells. In P. gingivalis, Leu-Asp- and Leu-Glu-MCA were shown to be primarily hydrolyzed by DPP11. If the same is also true for Bacteroides species, the inefficient Leu-Glu-MCA hydrolysis could be reproduced in these cells. Hence, we compared cellular activities toward the two substrates, and found that the ratio of hydrolysis of Leu-Glu-MCA per hydrolysis of Leu-Asp-MCA in P. gingivalis cells (0.496) was comparable to that of recombinant PgDPP11 (0.468) (Fig. 3). On the other hand, the ratio with $B$. fragilis cells was 0.17, which was larger than that of BfDPP11 (0.014), though substantially smaller than the ratio of PgDPP11 and P. gingivalis cells. Accordingly, it was confirmed that the ratio determined with bacterial cells principally reflected that of DPP11, though the differential extent was rather neutralized, possibly by the presence of minor Asp- and Glu-hydrolyzing activities in the cells. Similarly, the cellular ratios of B. thetaiotaomicron, B. uniformis, and B. dorei ranged at 0.09-0.23, which indicated that Bacteroides DPP11 commonly possesses very low activities for Leu-Glu-MCA.

\subsection{BfDPP11 3D Structural Modeling and Mechanism of its Inefficient Hydrolysis}

We performed 3D modeling of BfDPP11 using the structure of PeDPP11 (PDB code: 5JXK) [22], which indicated that BfDPP11 also has a bilobal architecture with an entirely helical domain capping the catalytic domain bearing a typical chymotrypsin double $\beta$-barrel fold. PeDPP11 3D structures in complexes with peptides (5JY0, 5JWG, 5JWI, 4XZY) and PgDPP11 showed that the S1 subsite is formed by $\operatorname{Arg}^{337}$, His ${ }^{649}, \mathrm{Thr}^{650}, \mathrm{Gly}^{652}, \mathrm{Asn}^{670}, \mathrm{Arg}^{673}, \mathrm{Gly}^{677}, \mathrm{Gly}^{680}$, $\mathrm{Asp}^{681}$, and Ser $^{691}$ (PgDPP11 numbering) [21, 22]. Among the ten residues, Gly ${ }^{652}$, Arg $^{673}$, Gly ${ }^{677}$, Gly ${ }^{680}$, and $\mathrm{Asp}^{681}$ are conserved, while the others, i.e., $\mathrm{Arg}^{337}{ }^{33 i}{ }^{649}, \mathrm{Thr}^{650}, \mathrm{Asn}^{670}$, and $\mathrm{Ser}^{691}$ are substituted 
by Asn, Asp, Asn, Gly, and Ala, respectively, in BfDPP11 (Fig. 4). Hence, we subsequently analyzed our model of BfDPP11 in order to structurally explain the inefficient cleavage of Leu-Glu-MCA.

The role of $\mathrm{Arg}^{337}$ of PgDPP11 has been extensively examined [21] and its substitution to Asn, Ala, and Gly was shown to lead to a significant increase in enzymatic activity. Thus, the Arg337Asn substitution of BfDPP11 could not explain the activity reduction of BfDPP11. Furthermore, $\mathrm{His}^{649}$ and $\operatorname{Ser}^{691}$ appear to be rather distant from the P1-position of a dipeptide, thus the influence of the two substitutions seems to be limited. In the rest two residues, $\mathrm{Thr}^{650}$ in PgDPP11 stabilizes the glutamic acid of incoming peptides via a hydrogen bond (Fig. 5). By superimposing BfDPP11 to PeDPP11 in a complex with Arg-Glu, we verified that the substitution $\mathrm{Thr}^{650}$ of PgDPP11 to Asn in BfDPP11 results in steric clashes according to the side chain orientation. In this manner, binding of incoming peptides with glutamic acid in the P1 position would be unfavorable in BfDPP11. Asn ${ }^{670}$ in PgDPP11 also stabilizes the Glu of incoming peptides via a hydrogen bond. However, $\mathrm{Asn}^{670}$ is replaced by Gly in BfDPP11, preventing the stabilization of the substrate Glu, resulting in decrease of the activity verified for Leu-Glu-MCA. On the other hand, $\mathrm{Val}^{678}$ in PgDPP11 is replaced by Leu in BfDPP11. Although $\mathrm{Val}^{678}$ does not directly form an S1 subsite, we speculate that the larger side chain of Leu of BfDPP11 influences the conformation of $\mathrm{Arg}^{673}$, which would explain the decrease in activity for Leu-Glu-MCA.

To verify our hypothesis, we expressed PgDPP11 and BfDPP11 with these substitutions (Fig. 6). The activity of BfDPP11 Asn650Thr displayed a 3.1-fold increase in Leu-Asp-MCA, with a further increase (8.1-fold) observed for the activity of Leu-Glu-MCA. As a result, the Glu/Asp ratio of the activity was increased by 2.6-fold in BfDPP11 Asn650Thr. In contrast, BfDPP11 Gly670Asn revealed little change. The third mutant, BfDPP11 Leu678Val, lost the activity for the two substrates, while the double mutation (Asn650Thr/Leu678Val) showed an intermediate amount of 
activity between those of the respective mutants. We also performed two reverse mutations with PgDPP11, Thr650Asn, and Asn670Gly. As a result, PgDPP11 Thr650Asn completely lost the activities for Leu-Asp-MCA and Leu-Glu-MCA, and PgDPP11 Asn670Gly revealed extremely low activities for those $(0.53 \%$ and $0.18 \%$, respectively). Therefore, all mutations except for Gly670Asn in BfDPP11 had effects on the activities of BfDPP11 or PgDPP11. In particular, it should be emphasized that mutual exchanges between $\operatorname{Thr}^{650}$ of PgDPP11 and Asn ${ }^{650}$ of BfDPP11 partially mimicked the change between PgDPP11 and BfDPP11.

When the amino acid substitutions at positions 650,670 , and 678 were reexamined in the sequences, only $\mathrm{Thr}^{650}$ and $\mathrm{Asn}^{650}$ were found to be strictly distributed in non-Bacteroides and Bacteroides DPP11, respectively (Fig. 4). In contrast, residues at positions 670 and 678 had variations even among listed DPP11s, findings compatible with our biochemical data (Fig. 6). Nevertheless, it should be noted that the Leu-Glu-MCA/Leu-Asp-MCA ratio of PgDPP11 (0.468) was still much greater than that of BfDPP11 Asn650Thr (0.038), suggesting that other factors may be involved in this phenomenon.

\section{Discussion}

To date, the enzymatic properties of both DPP7 and DPP11 have been characterized in only three bacterial species, i.e., P. gingivalis [1, 2], P. endodontalis [2, 28], and C. gingivalis [20], while either one of DPP7 or DPP11 has been characterized in B. fragilis, B. vulgatus, F. psychrophilum, S. putrefaciens [20], P. mexicana [18], and Capnocytophaga canimorsus [29]. At present, though their homologues are widely distributed among eubacteria, most S46-family members are tentatively classified by their sequence similarity to authentic S46 members of $P$. gingivalis, and their enzymatic characteristics remain to be elucidated. 
In the present study, we confirmed the validity of the classification criteria for the S1 amino acid residues $\mathrm{Gly}^{673} / \mathrm{Arg}^{673}$ for identification of DPP7 and DPP11, and found that classification of the S46 peptidase based on full-length similarity misleads the annotation. For example, putative Bacteroides DPP11 genes (BF9343_0130, Bovatus_00118), which exhibit a higher similarity to PgDPP11 than PgDPP7 but carry Gly ${ }^{673}$, encode DPP7. Similarly, Hack et al. [29] recently reported that the gene Ccan_08540 from C. canimorsus encoding a DPP with Gly ${ }^{673}$ convincingly exhibited DPP7 activity, though the gene was tentatively annotated as DPP11.

We also demonstrated that Bacteroides DPP11 exhibited decreased hydrolyzing activity, especially toward the substrate with P1-position Glu. Accordingly, there are three types of DPP11 based on substrate specificity, with the first being $\operatorname{Arg}^{673}$ possessing greater preference for the P1-position Asp of a substrate (Asp>Glu type) represented by P. gingivalis, the second Shewanella type DPP11 possessing $\mathrm{Ser}^{673}$ and $\mathrm{Lys}^{684}$, and exhibiting a higher Glu preference (Glu>Asp type), and the third Bacteroides type carrying $\operatorname{Arg}^{673}$, which is much less potent for Glu (Asp $>>$ Glu). The present and previous 3D structural modeling and substitution analyses revealed that alterations in the P1-position preference among the three types appeared to be mediated by a few amino acid substitutions. Hence, essential amino acid residues can be used for simple discrimination of the 3 DPP11 types; $\mathrm{Thr}^{650}$ and $\mathrm{Arg}^{673}$ for the Asp $>$ Glu type, $\mathrm{Asn}^{650}$ and $\operatorname{Arg}^{673}$ for the Asp $>>$ Glu type, and $\mathrm{Thr}^{650}, \mathrm{Ser}^{673}$, and Lys ${ }^{684}$ for the Glu>Asp type.

The present findings also indicate the existence of the third group in S46 peptidase, which has been annotated simply as DPP7 in Bacteroides species. Although these putative DPP7 genes, such as BF9343_0130 and Bovatus_03382, showed more than 40\% sequence similarity to PgDPP7 and carrying Gly ${ }^{673}$, they showed no hydrolytic activity for the dipeptidyl MCA currently available in our laboratory. In vitro mutagenesis analysis further indicated that BF9343_0130 and Bovatus_03382 are substantially distant from DPP7 as well as DPP11. The possibility of a 
pseudogene cannot be excluded in this entity, however, general distribution of the third S46 gene in the genus Bacteroides strongly suggests its physiological significance. Among previously reported DPPs, DPPX/DPP10, possessing structural similarity to DPP4, has no peptidase activity due to a point mutation in its active site [30]. In this analogy, the third group of S46 members might have no peptidase activity. A further study should be needed for characterization of this group.

Taken together, we propose that there are three members of the S46 family, DPP7, DPP11, and an uncharacterized group. We previously reported that the phylogenic tree of the S46 family, with construction based on the C-terminal conserved region $\left(\mathrm{Ser}^{571}-\mathrm{Leu}^{700}\right)$ rather than the tree based on the full-length form, matches the distribution of DPP7 and DPP11 [20]. Since the present analysis demonstrated the presence of Bacteroides-type DPP11 and the third S46 group members, we reexamined the phylogenic tree. As shown in Fig. 7, S46 peptidases have two branches of DPP7, one that includes Porphyromonas and Bacteroides species, and the other with Shewanella and Xanthomonas species. DPP7 and DPP11 of the genus Bacteroides form branching links independent of other DPP7 and DPP11 groups, respectively, and the third group is arranged at the opposite end from Bacteroides DPP7. As a result, classification of most parts of S46 peptidases was ascertained in the present study, except for Group 1, which is composed of an unassigned S46 peptidase possessing Gly ${ }^{673}$ or $\mathrm{Ser}^{673}$. The members of this branch seem to have sequence variations, and compose a mixture of DPP7 and DPP11.

In P. gingivalis, the two S46-family genes are localized at separate loci as PGN_0607 (DPP11) and PGN_1479 (DPP7). In contrast, two of the three S46 family genes of Bacteroides species are adjacently located, i.e., BF9343_2924/DPP7 and BF9343_2925/DPP11 in B. fragilis are one-base

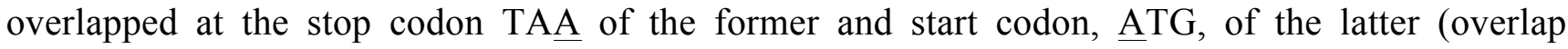
underlined). Moreover, the genes of Bovatus_00117/BfDPP11 and Bovatus_00118/BfDPP7 in $B$. ovatus are directly connected without a gap. Hence, these two genes form an operon. These 
observations suggest that the gene duplication possibly occurred in an S46-family ancestor gene, then became to the DPP7 and DPP11 genes. In contrast, the third S46-family gene is separately localized from the other two S46-family genes on the genome, and amino acid identity between BfDPP7 and BfDPP11 (42.8\%) was found to be higher than that between BF9343_0130 (unidentified group) and BfDPP7 (37.5\%), as well as that between BF9343_0130 (unidentified group) and BfDPP11 (35.7\%) (Table 3).

Finally, it is important to emphasize that newly synthesized Phe-Leu- and Leu-Leu-MCA are more potent DPP7 substrates compared to Lys-Ala- and Met-Leu-MCA, which were previously used. These substrates should be valuable for examining the DPP7 activities of oral and intestinal bacterial cells. However, we would like to note that $k_{\text {cat }} / K_{\mathrm{m}}$ of PgDPP7 for Leu-Leu-MCA was still at a one to 34 ratio of that of $P$. gingivalis DPP4 for Gly-Pro-MCA [15] and one to 14.5 of that of PgDPP11 for Leu-Asp-MCA. We suppose that this may be due to the necessity in DPP7 for exertion of the broad specificity. In other words, in contrast to DPP4 and DPP11, which are highly specific for Pro and Asp/Glu, respectively, DPP7 is able to accept P1-position hydrophobic residues with various residual groups. This redundancy of DPP7 may be achieved by a sacrifice in $k_{\text {cat }} / K_{\mathrm{m}}$ value.

\section{Acknowledgments}

This work was supported by JSPS KAKENHI Grants Numbers JP15K11047, JP16K11481, and JP17K17336. A.G. Bezerra was supported by the Vienna International Postdoctoral Program (VIPS). 


\section{Author contributions}

Author contributions for this study were as follows: conceptualization (T.K.N.), methodology (T.K.N., Y.O-N., A.G.B., T.O.), investigation (H.N., T.F., A.G.B., Y.O-N., T.K.N. T.O.), writing of original draft (T.K.N.), review and editing (Y.O-N., T.K.N., A.G.B.), and funding acquisition (T.K.N., Y.O-N., N.H.). 


\section{REFERENCES}

[1] A. Banbula, J. Yen, A. Oleksy, P. Mak, M. Bugno, J. Travis, J. Potempa, Porphyromonas gingivalis DPP-7 represents a novel type of dipeptidylpeptidase. J. Biol. Chem. 276 (2001) 6299-6305.

[2] Y. Ohara-Nemoto, Y. Shimoyama, S. Kimura, A. Kon, H. Haraga, T. Ono, T.K. Nemoto, Aspand Glu-specific novel dipeptidyl peptidase 11 of Porphyromonas gingivalis ensures utilization of proteinaceous energy sources. J. Biol. Chem. 286 (2011) 38115-38127.

[3] A.L. Griffen, M.R. Becker, S. R. Lyons, M.L. Moeschberger, E.J. Leys, Prevalence of Porphyromonas gingivalis and periodontal health status. J. Clin. Microbiol. 36 (1998) 32393242.

[4] S.C. Holt, J.L. Ebersole, Porphyromonas gingivalis, Treponema denticola, and Tannerella forsythia: the 'red complex', a prototype polybacterial pathogenic consortium in periodontitis. Periodontology 200038 (2005) 72-122.

[5] T.J.M. van Steenbergen, A.J. van Winkelhoff, D. Mayrand, D. Grenier, J. de Graaff, Bacteroides endodontalis sp. nov., an asaccharolytic black-pigmented Bacteroides species from infected dental root canals. Int. J. Syst. Bacteriol. 34 (1984) 118-120.

[6] A.J. van Winkelhoff, N. Kippuw, J. de Graaff, Further characterization of Bacteroides endodontalis, an asaccharolytic black-pigmented Bacteroides species from the oral S cavity. J. Clin. Microbiol. 22 (1985) 75-79.

[7] Z. Chen, J. Potempa, A. Polanowski, M. Wikstrom, J. Travis, Molecular cloning and structural characterization of the Arg-gingipain proteinase of Porphyromonas gingivalis. Biosynthesis as a proteinase-adhesin polyprotein. J. Biol. Chem. 267 (1992) 18896-18901.

[8] H.N. Shah, S.E. Gharbia, D. Kowlessur, E. Wilkie, K. Brocklehurst, Isolation and characterization of gingivain, a cysteine proteinase from Porphyromonas gingivalis strain W83. Biochem. Soc. Tran. 18 (1990) 578-579.

[9] Y. Abiko, M. Hayakawa, S. Murai, H. Takiguchi, Glycylprolyl dipeptidylaminopeptidase from Bacteroides gingivalis. J. Dent. Res. 64 (1985) 106-111.

[10] A. Banbula, M. Bugno, J. Goldstein, J. Yen, D. Nelson, J. Travis, J. Potempa, Emerging family of proline-specific peptidases of Porphyromonas gingivalis: purification and characterization of serine dipeptidyl peptidase, a structural and functional homologue of mammalian prolyl dipeptidyl peptidase IV. Infect. Immun. 68 (2000) 1176-1182. 
[11] Y. Ohara-Nemoto, S. M. Rouf, M. Naito, A. Yanase, F. Tetsuo, T. Ono, T. Kobayakawa, Y. Shimoyama, S. Kimura, K. Nakayama, K. Saiki, K. Konishi, T.K. Nemoto, Identification and characterization of prokaryotic dipeptidyl-peptidase 5 from Porphyromonas gingivalis. J. Biol. Chem. 289 (2014) 5436-5448.

[12] A. Banbula, P. Mak, M. Bugno, J. Silberring, A. Dubin, D. Nelson, J. Travis, J. Potempa, Prolyl tripeptidyl peptidase from Porphyromonas gingivalis. A novel enzyme with possible pathological implications for the development of periodontitis. J. Biol. Chem. 274 (1999) 9246-9252.

[13] K. Ito, Y. Nakajima, Y. Xu, N. Yamada, Y. Onohara, T. Ito, T., Matsubara, F., Kabashima, T., Nakayama, K., and Yoshimoto, T. (2006) Crystal structure and mechanism of tripeptidyl activity of prolyl tripeptidyl aminopeptidase from Porphyromonas gingivalis. J. Mol. Biol. 362 (2006) 228-240.

[14] M. Kiyama M. Hayakawa, T. Shiroza, S. Nakamura, A. Takeuchi, Y. Masamoto, Y. Abiko, Sequence analysis of the Porphyromonas gingivalis dipeptidyl peptidase IV gene. Biochim. Biophys. Acta 1396 (1998) 39-46.

[15] Y. Ohara-Nemoto, M. Nakasato, Y. Shimoyama, T.T. Baba, T. Kobayakawa, T. Ono, T. Yaegashi, S. Kimura, T.K. Nemoto, Degradation of incretins and modulation of blood glucose levels by periodontopathic bacterial dipeptidyl peptidase 4. Infect. Immun. 85 (2017) e00277-17.

[16] T.K. Nemoto, Y. Ohara-Nemoto, G.A. Bezerra, Y. Shimoyama, S. Kimura, Porphyromonas gingivalis periplasmic novel exopeptidase, acylpeptidyl oligopeptidase, releases $\mathrm{N}$-acylated di- and tripeptides from oligopeptides. J. Biol. Chem. 291 (2016) 5913-5925.

[17] T.K. Nemoto, Y. Ohara-Nemoto, Exopeptidases and gingipains in Porphyromonas gingivalis as prerequisites for its amino acid metabolism Jpn. Dental Sci. Rev. 52 (2016) 22-29.

[18] Y. Suzuki, Y. Sakamoto, N. Tanaka, H. Okada, Y. Morikawa, W. Ogasawara, Identification of the catalytic triad of family S46 exopeptidases, closely related to clan PA endopeptidases. Sci. Rep. 4 (2014) 4292.

[19] N.D. Rawlings, M. Waller, A.J. Barrett, A. Bateman, MEROPS: the database of proteolytic enzymes, their substrates and inhibitors. Nucleic Acids Res. 42 (2014) D503-D509.

[20] S.M. Rouf, Y, Ohara-Nemoto, T. Hoshino, T. Fujiwara, T. Ono, T.K. Nemoto, Discrimination based on Gly and Arg/Ser at position 673 between dipeptidyl-peptidase (DPP) 7 and DPP11, widely distributed DPPs in pathogenic and environmental Gram-negative bacteria. Biochimie 95 (2013) 824-832. 
[21] Y. Sakamoto, Y. Suzuki, Y. Iizuka, C. Tateoka, S. Roppongi, M. Fujimoto, K. Inaka, H. Tanaka, M. Yamada, K. Ohta, H. Gouda, T, Nonaka, Y. Ogasawara, N. Tanaka, Structural and mutational analyses of dipeptidyl peptidase 11 from Porphyromonas gingivalis reveal the molecular basis for strict substrate specificity. Sci. Rep. 5 (2015) 11151.

[22] G. A. Bezerra, Y. Ohara-Nemoto, I. Cornaciu, S. Fedosyuk, G. Hoffmann, A. Round, J. A. Márquez, T. K. Nemoto, K. Djinović-Carugo, Bacterial protease uses distinct thermodynamic signatures for substrate recognition Sci Rep. 7 (2017) 2848.

[23] S.M.A. Rouf, Y. Ohara-Nemoto, T. Ono, Y. Shimoyama, S. Kimura, T.K. Nemoto, Phenylalanine664 of dipeptidyl peptidase (DPP) 7 and phenylalanine671 of DPP11 mediate preference for P2-position hydrophobic residues of a substrate. FEBS Open Bio, 3 (2013) $177-181$.

[24] L.A. Kelley, S. Mezulis, C.M. Yates, M.N. Wass, M. J. Sternverg, The Phyre2 web portal for protein modeling, prediction and analysis. Nat. Protoc. 10 (2015) 845-858.

[25] J.D. Thompson, T.J. Gibson, F. Plewniak, F. Jeanmougin, D.G. Higgins, The CLUSTAL_X Windows interface: flexible strategies for multiple sequence alignment aided by quality analysis tools, Nucleic Acids Res. 25 4876e (1997) 4882.

[26] N. Saitou, M. Nei, The neighbor-joining method: a new method for reconstructing phylogenetic trees, Mol. Biol. Evol. 4 (1997) 406e425.

[27] K. Tamura, D. Peterson, N. Peterson, G. Stecher, M. Nei, S. Kumar, MEGA5: molecular evolutionary genetics analysis using maximum likehood, evolutionary distance, and maximum parsimony methods, Mol. Biol. Evol. 28 (2011) 2731-2739.

[28] H. Nishimata, Y. Ohara-Nemoto, T.T. Baba, T. Hoshino, T. Fujiwara, Y. Shimoyama, S. Kimura T.K. Nemoto, Identification of dipeptidyl-peptidase (DPP) 5 and DPP7 in Porphyromonas endodontalis, distinct from those in Porphyromonas gingivalis PLoS One 9 (2014) e114221.

[29] K. Hack, F. Renzi, E. Hess, F. Lauber, J. Douxfils, J.M. Dogné, G.R. Cornelis, Inactivation of human coagulation factor $\mathrm{X}$ by a protease of the pathogen Capnocytophaga canimorsus. J. Thromb. Haemost. 15 (2017) 487-499.

[30] S.Y. Qi, P.J. Riviere, J. Trojnar, J.L. Junien, K.O. Akinsanya, Cloning and characterization of dipeptidyl peptidase 10, a new member of an emerging subgroup of serine protease. Biochem. J. 2003, 37 (2003) 179-189. 


\section{Table 1}

Primers and plasmids used in this study. Restriction and mutated sites are shown by italics and underlining, respectively.

\begin{tabular}{|c|c|c|}
\hline Name & Sequence & $\begin{array}{l}\text { Plasmid (substituted } \\
\text { nucleotides) }\end{array}$ \\
\hline 5BF9343_0130N2Bam & TTTATAGGATCCAATAAAATGAAAGTGATTA & \multirow[t]{2}{*}{ pTrcHis2BF0130 } \\
\hline 3BF9343_0130G725Bam & GTGATGGGATCCCCCCTCTATTTTCAGTTCT & \\
\hline 5BF9343_2925M2Bam & ATATAGGATCCATGAAAAGAAACTTATTATC & \multirow[t]{2}{*}{ pQE60BF2925 } \\
\hline 3BF9343_2925E721Bam & TCTGTGGATCCTTCTTCTACAATATCCAGCT & \\
\hline 5BVU_2252D20 & GACGAGGGGATGTGGATGCTGACTGA & \multirow[t]{2}{*}{ pTrcHis2BV2252 } \\
\hline 3BVU_2252E728 & TTCCACAATGGTCATTTCATCTACCA & \\
\hline 5BF9343_0130G673R & CGAAACTGGGAAGCCATGAGCAG & \multirow{2}{*}{$\begin{array}{l}\text { pTrcHis2BF0130Gly673Ar } \\
\mathrm{g}(\underline{\mathrm{G} G A}>\underline{\mathrm{CGA}})\end{array}$} \\
\hline 3BF9343_0130D672 & ATCGAACGCCAATCCCAGCAATT & \\
\hline 5BF9343_2924G673R & CGTAACTGGGAATCACTGAGCGG & \multirow{2}{*}{$\begin{array}{l}\text { pTrcHis2BF2924Gly673Ar } \\
\mathrm{g}(\underline{\mathrm{G} G T}>\underline{\mathrm{CGT}})\end{array}$} \\
\hline 3BF9343_2924D672 & ATCGAATGCACAACCGATCAATT & \\
\hline 5 PgDPP11T650N & AATACCGGCGGCAACTCAGGCAGTCCGGTC & \multirow{2}{*}{$\begin{array}{l}\text { pQPgDPP11Thr650Asn } \\
(\mathrm{ACA}>\mathrm{AAT})\end{array}$} \\
\hline 3 PgDPP11H649 & ATGTGTGGTGGCACAAAAGGCGACAGG & \\
\hline 5PgDPP11N670G & GGATTCGATCGTAACTGGGAGGGAGTCGG & \multirow{2}{*}{$\begin{array}{l}\text { pQPgDPP11Asn670Gly } \\
(\underline{\text { AAC }>\text { GGA) }}\end{array}$} \\
\hline 3PgDPP11L669 & GAGACCGATCAGTTCGCCGTTGGCATTCA & \\
\hline $5 \mathrm{BfDPP} 11 \mathrm{~N} 650 \mathrm{~T}$ & ACAACAGGAGGAAATTCCGGAAGCC & \multirow{2}{*}{$\begin{array}{l}\text { pQBF2925Asn650Thr } \\
\text { (A푸 }>\text { A } \underline{\text { A }} \text { ) }\end{array}$} \\
\hline 3BfDPP11D649 & GTCTGTATTGACAATGAAGCAAACAGGCA & \\
\hline 5BfDPP11G670N & AACTTCGATCGTAATTATGAAGGCCTGAC & \multirow{2}{*}{$\begin{array}{l}\text { pQBF2925Gly670Asn } \\
(\underline{\text { GGA }}>\underline{A A C})\end{array}$} \\
\hline 3BfDPP11T669 & GTCCCGATCAATTGCCCTTTTCCATTGA & \\
\hline 5BfDPP11L678V & GTCACAGGAGACATTGCTTTCCGG & \multirow{2}{*}{$\begin{array}{l}\text { pQBF2925Leu678Val } \\
\text { (CTG }>\text { GTC) }\end{array}$} \\
\hline 3BfDPP11G677 & GCCTTCATCATTACGATCGAATCCGGTC & \\
\hline
\end{tabular}


Table 2

Enzymatic parameters of $P$. gingivalis and $B$. fragilis DPPs.

\begin{tabular}{|c|c|c|c|c|c|}
\hline Dipeptidyl & MCA & & & $k_{\text {cat }} / K_{\mathrm{m}}$ & \\
\hline peptidase & peptide & $k_{\text {cat }}\left(\sec ^{-1}\right)$ & $K_{m}(\mu \mathrm{M})$ & $\left(\mu \mathrm{M}^{-1} \sec ^{-1}\right)$ & Reference \\
\hline \multicolumn{6}{|c|}{ P. gingivalis } \\
\hline DPP4 & Gly-Pro- & $121,752 \pm 2,974$ & $94.9 \pm 2.1$ & $1,283.3 \pm 22.5$ & {$[15]$} \\
\hline DPP5 & Lys-Ala- & $1,948 \pm 165$ & $185 \pm 21$ & 10.5 & [11] \\
\hline \multirow[t]{4}{*}{ DPP7 } & Met-Leu- & $394 \pm 79$ & $39.6 \pm 16.0$ & $10.6 \pm 2.5$ & {$[23]$} \\
\hline & Leu-Leu- & $753.9 \pm 1.9$ & $20.0 \pm 0.6$ & $37.7 \pm 1.1$ & this study \\
\hline & Phe-Leu- & $2,044 \pm 254$ & $134.2 \pm 18.9$ & $15.3 \pm 0.6$ & this study \\
\hline & His-Leu- & $1,137 \pm 203$ & $648 \pm 136$ & $1.73 \pm 0.05$ & this study \\
\hline \multirow[t]{2}{*}{ DPP11 } & Leu-Asp- & $10,707 \pm 140$ & $19.5 \pm 0.4$ & $547.4 \pm 6.3$ & [17] \\
\hline & Leu-Glu- & $13,587 \pm 577$ & $80.9 \pm 5.7$ & $167.7 \pm 5.3$ & this study \\
\hline \multicolumn{6}{|l|}{ B. fragilis } \\
\hline \multirow[t]{3}{*}{ DPP11 } & Leu-Asp- & $7,379 \pm 994$ & $183.3 \pm 41.1$ & $40.9 \pm 4.4$ & this study \\
\hline & Leu-Glu- & $25,865 \pm 2,473$ & $29,710 \pm 1,629$ & $0.87 \pm 0.04$ & this study \\
\hline & & $\mathrm{x} 10^{3}$ & $\mathrm{x} 10^{3}$ & & \\
\hline
\end{tabular}




\section{Table 3}

Essential amino acids, sequence identity, and identification of Bacteroides S46 DPP members.

\begin{tabular}{|c|c|c|c|c|c|c|c|c|c|c|c|c|}
\hline \multirow[b]{3}{*}{ Species } & \multirow[b]{3}{*}{ Gene } & \multirow[b]{3}{*}{ MEROPS ID } & \multirow[b]{3}{*}{ Annotation $^{\mathrm{a}}$} & \multirow[b]{3}{*}{$\mathrm{Xaa}^{673}$} & \multicolumn{6}{|c|}{ Similarity $\quad(\%)$} & \multirow[b]{3}{*}{ Identification } & \multirow{3}{*}{ Reference } \\
\hline & & & & & 2924 & 2925 & 00118 & 03382 & & & & \\
\hline & & & & & (BfDPP7) & (BfDPP11) & (BoDPP7) & $(\text { Bo-third })^{b}$ & PgDPP7 & PgDPP11 & & \\
\hline \multirow[t]{3}{*}{ B. fragilis } & 0130 & 039992 & DPP7 & Gly & 37.5 & 35.7 & 37.1 & 76.9 & 43.0 & 34.2 & third group $^{b}$ & this study \\
\hline & 2924 & 039993 & DPP11 & Gly & 100 & 45.8 & 84.3 & 38.2 & 39.5 & 44.7 & DPP7 & [20] \\
\hline & 2925 & 039991 & DPP11 & Arg & & 100 & 44.9 & 35.3 & 37.2 & 45.1 & DPP11 & this study \\
\hline \multirow[t]{2}{*}{ B. ovatus } & 00118 & 0109242 & DPP11 & Gly & & & 100 & 37.6 & 37.5 & 44.5 & DPP7 & this study \\
\hline & 03382 & 0109141 & DPP7 & Gly & & & & 100 & 40.5 & 34.9 & third group $^{b}$ & this study \\
\hline$P$. gingivalis & 0607 & 0034628 & DPP11 & Arg & & & & & & 100 & DPP11 & [2] \\
\hline
\end{tabular}

'Based on comparison of full-length amino acid sequence (MEROPS). Genomic sequence data shown are from B. fragilis ATCC 25285 (BF9343), B. ovatus ATCC 8483 (Bovatus), and P. gingivalis ATCC 33277 (PGN). 'See text in detail. 
A

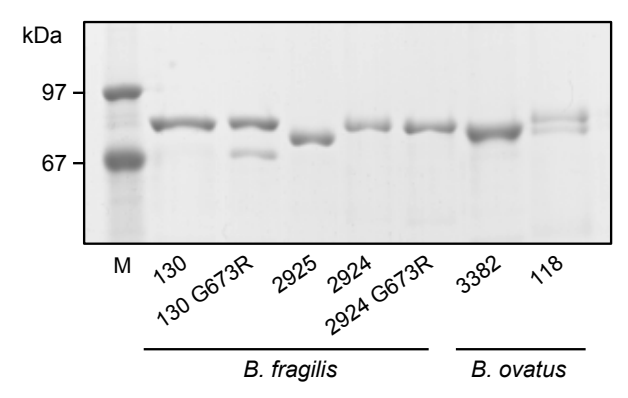

B

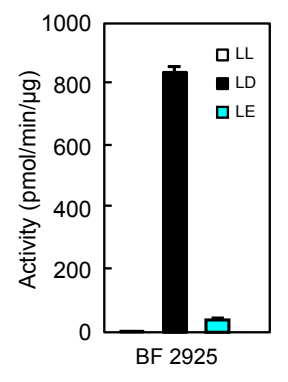

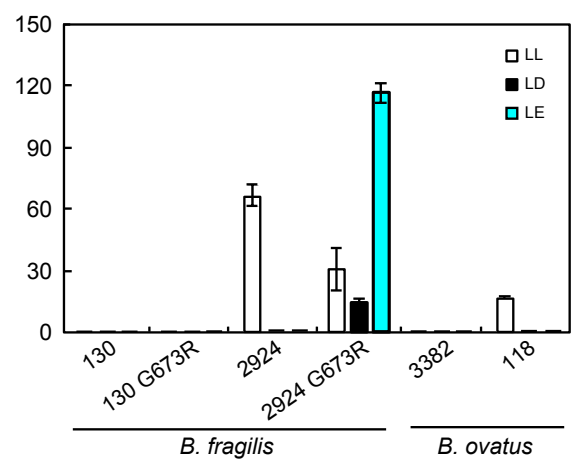

Fig. 1. Activities of B. fragilis and B. ovatus DPPs in S46 family.

(A) Putative S46 peptidase genes from B. fragilis (130, BF9343_0130; 2925, BF9343_2925; 2924, BF2925_2924), B. ovatus (3382, Bovatus_03382; 118, Bovatus_03382), and Gly673Arg mutants of BF9343_0130 and BF2925_2924 were expressed. Purified DPPs and their mutants $(0.4 \mu \mathrm{g})$ were separated on SDS-PAGE. M, low-molecular-weight marker. (B) DPP activities were measured with Leu-Leu-, Leu-Asp-, and Leu-Glu-MCA. Values are shown as the mean \pm S.D. $(\mathrm{n}=3)$. 
A

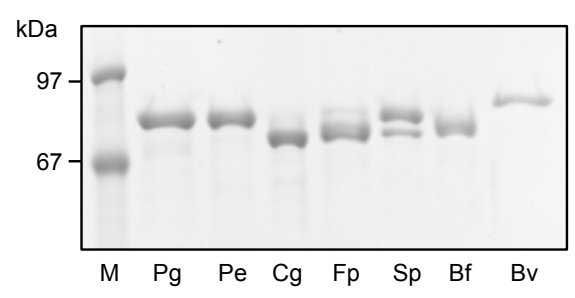

C

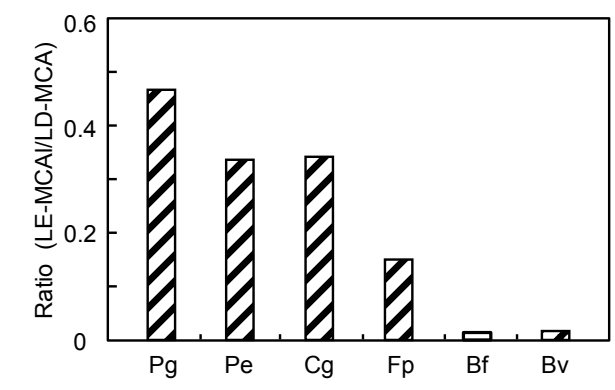

C
B

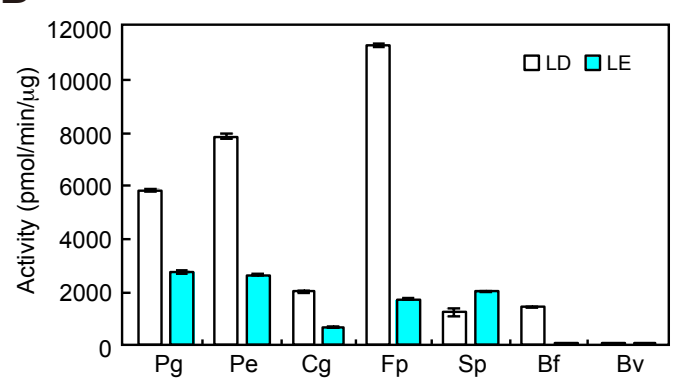

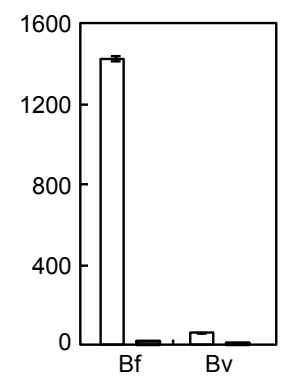

Fig. 2. Expression and characterization of three types of DPP11s.

(A) Recombinant forms of DPP11 (0.4 $\mu \mathrm{g})$ from P. gingivalis (Pg), P. endodontalis (Pe), C. gingivalis $(\mathrm{Cg})$, and F. psychrophilum (Fp), which carry $\mathrm{Arg}^{673}$, that from S. putrefaciens $(\mathrm{Sp})$ with $\operatorname{Ser}^{673}$ and $\mathrm{Lys}^{680}$, and putative DPP11 from B. fragilis (Bf) (BF9343_2925) and B. vulgatus (Bv) (BVU_2252) with $\mathrm{Arg}^{673}$ were separated on SDS-PAGE. M, low-molecular-weight marker. (B) Activities of recombinant DPP11s were measured with Leu-Asp- and Leu-Glu-MCA. Values are shown as the mean \pm S.D. $(\mathrm{n}=3)$. Data for B. fragilis (Bf) and B. vulgatus (Bv) DPP11 are expanded in the right panel. (C) Ratio of activity for Leu-Glu-MCA to that for Leu-Asp-MCA is plotted, except for S. putrefaciens DPP11. 
A

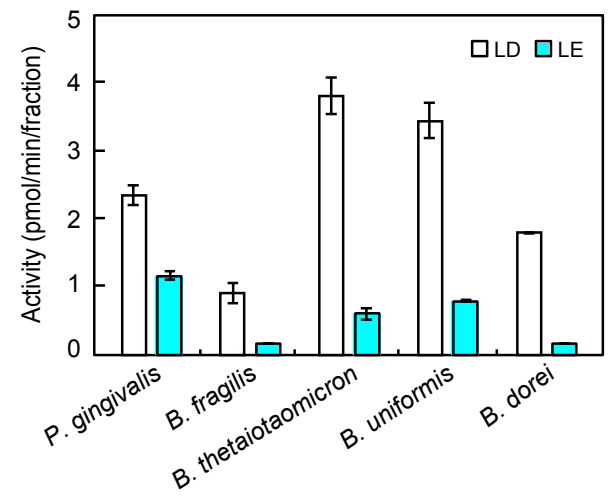

B

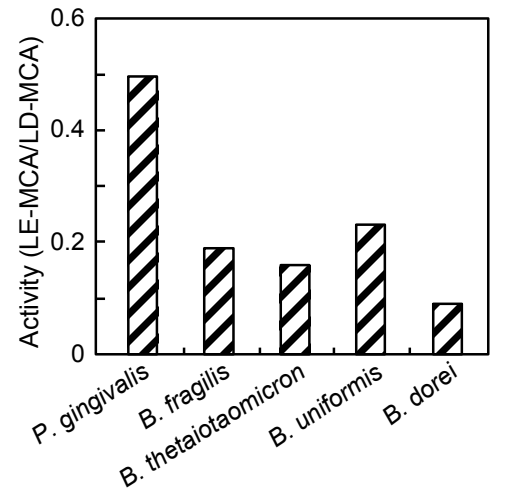

Fig. 3. DPP11 activity in Bacteroides cells.

(A) Hydrolyses for Leu-Asp- and Leu-Glu-MCA were determined using bacterial cell suspensions $\left(4 \mu \mathrm{l}, \mathrm{A}_{600}=5\right)$. Values are shown as the mean \pm S.D. $(\mathrm{n}=3)$. (B) Ratio of activity for Leu-Glu-MCA to that for Leu-Asp-MCA is plotted. P. gingivalis ATCC 33277, B. fragilis YCH10, B. thetaiotaomicron KYU12, B. uniformis ATCC 8492, and B. dorei JCM 13471 cells were used. 


P. gingivalis
P. endodontalis
F. psychrophilum
C. gingivalis
S. putrefaciens
B. fragilis
B. vulgatus
B. thetaiotaomicron
B. uniformis
B. caccae
B. finegoldii
B. clarus
B. eggerthii
B.coprocola

Fig. 4. Comparison of amino acid sequences around essential serine residue in DPP11s.

The amino acid sequences at $\mathrm{Met}^{640}-\mathrm{Asp}^{681}$ of 7 DPP11s expressed in this study are shown at the top, 2 Bacteroides DPP11 are shown at the middle, of which cell activities were measured, and 5 other Bacteroides DPP11s are shown at the bottom, and were compared. Identical amino acid residues are indicated by asterisks and those with a conserved property are indicated by colons under the sequences. The $\beta$-strand and 3/10-helix regions are indicated at the bottom. Ser ${ }^{655}$ composing the charge relay system, and $\mathrm{Arg}^{673}$ and Lys ${ }^{680}$, essential for the P1 Asp/Glu specificity of DPP11, are shown in red. His ${ }^{649}, \mathrm{Thr}^{650}, \mathrm{Asn}^{670}$, and Val ${ }^{678}$ (blue) of PgDPP11 are substituted by other amino acids (green) in Bacteroides, and other bacterial DPP11s. Among them, $\mathrm{Thr}^{650}, \mathrm{Asn}^{670}$, and $\mathrm{Val}^{678}$ are potentially responsible for the alteration in the activity in Bacteroides DPP11 (see text). 
A

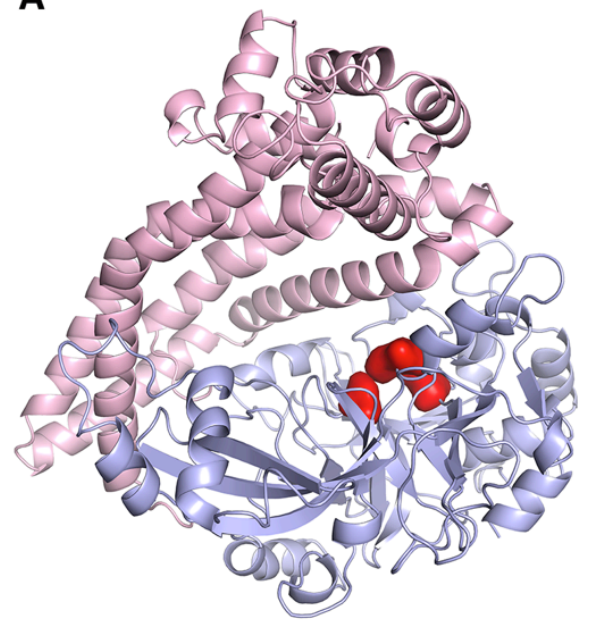

C

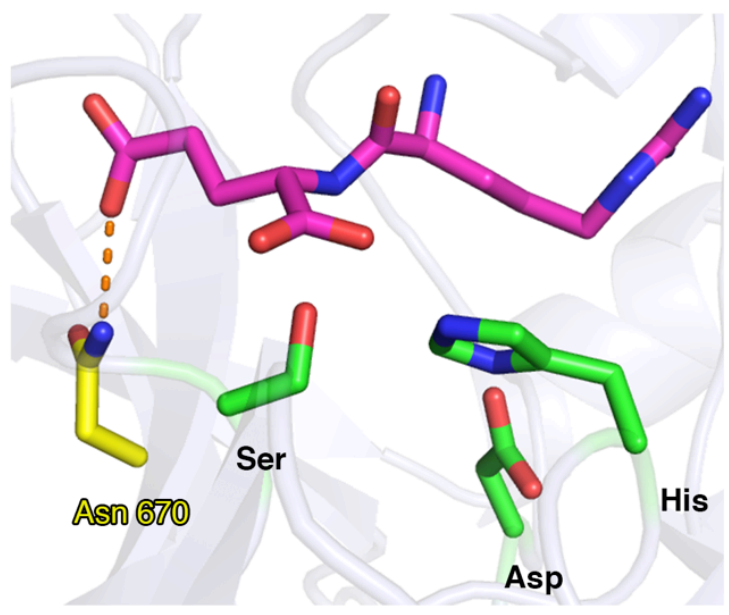

B

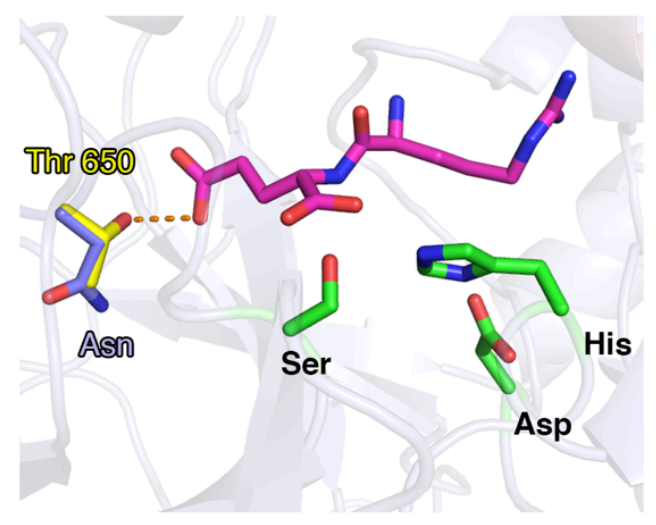

D

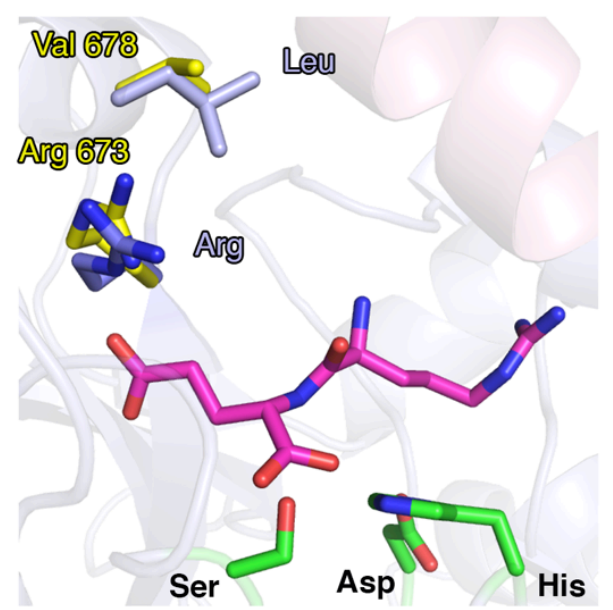

Fig. 5. 3D-homology model of BfDPP11.

(A) The catalytic fold in the overall structure of BfDPP11 is represented in blue and the helical fold in pink. The location of the catalytic triad is shown in red. (B-D) BfDPP11 is superimposed to PeDPP11 in the complex with Arg-Glu (magenta). The catalytic triad (His ${ }^{85}, \mathrm{Asp}^{227}, \mathrm{Ser}^{655}$ ) is shown as green sticks. Nitrogen and oxygen are represented by blue and red, respectively. (B) $\mathrm{Thr}^{650}$, (C) $\mathrm{Asn}^{670}$, and (D) $\mathrm{Val}^{678}$ are substituted by Asn, Gly, and Leu (yellow), respectively, in BfDPP11. All amino acid numbers are indicated as those of PgDPP11. 

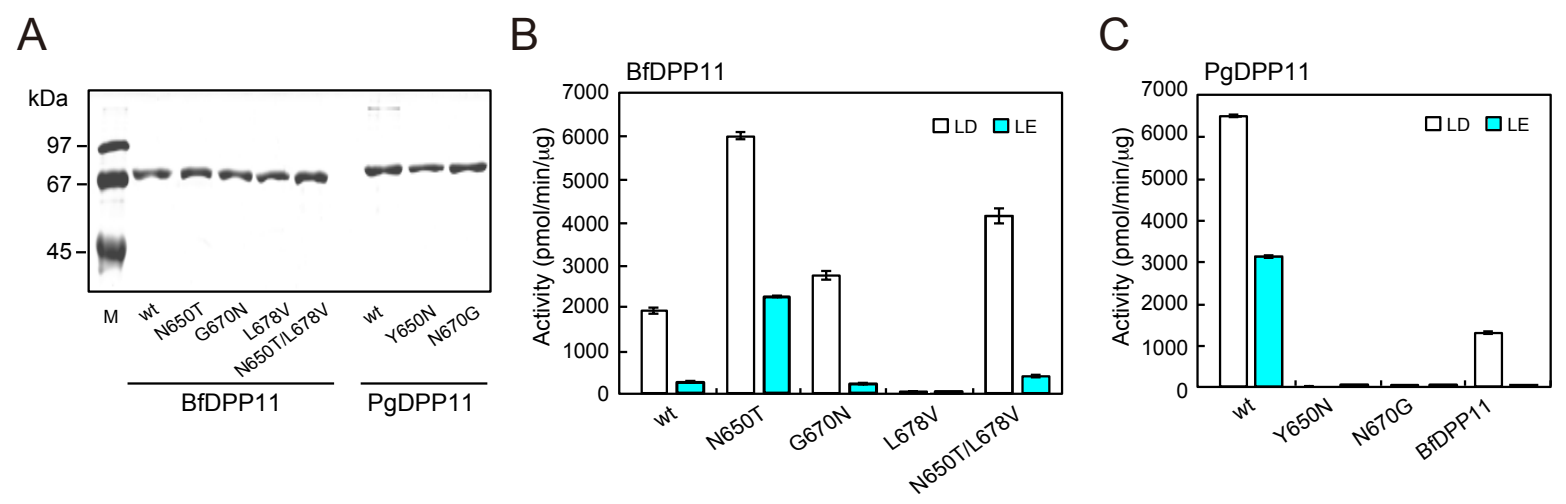

Fig. 6. Effect of in vitro mutagenesis of PgDPP11 and BfDPP11 at positions 650, 670, and 678. (A) Purified proteins $(0.4 \mu \mathrm{g})$ of BfDPP11 and PgDPP11, and their mutants were separated on SDS-PAGE. M, low-molecular-weight marker. (B) Activities of BfDPP11 and its mutants, and (C) those of PgDPP11 and its mutants were determined with Leu-Asp- and Leu-Glu-MCA. Values are shown as the mean \pm S.D. $(n=3)$. 


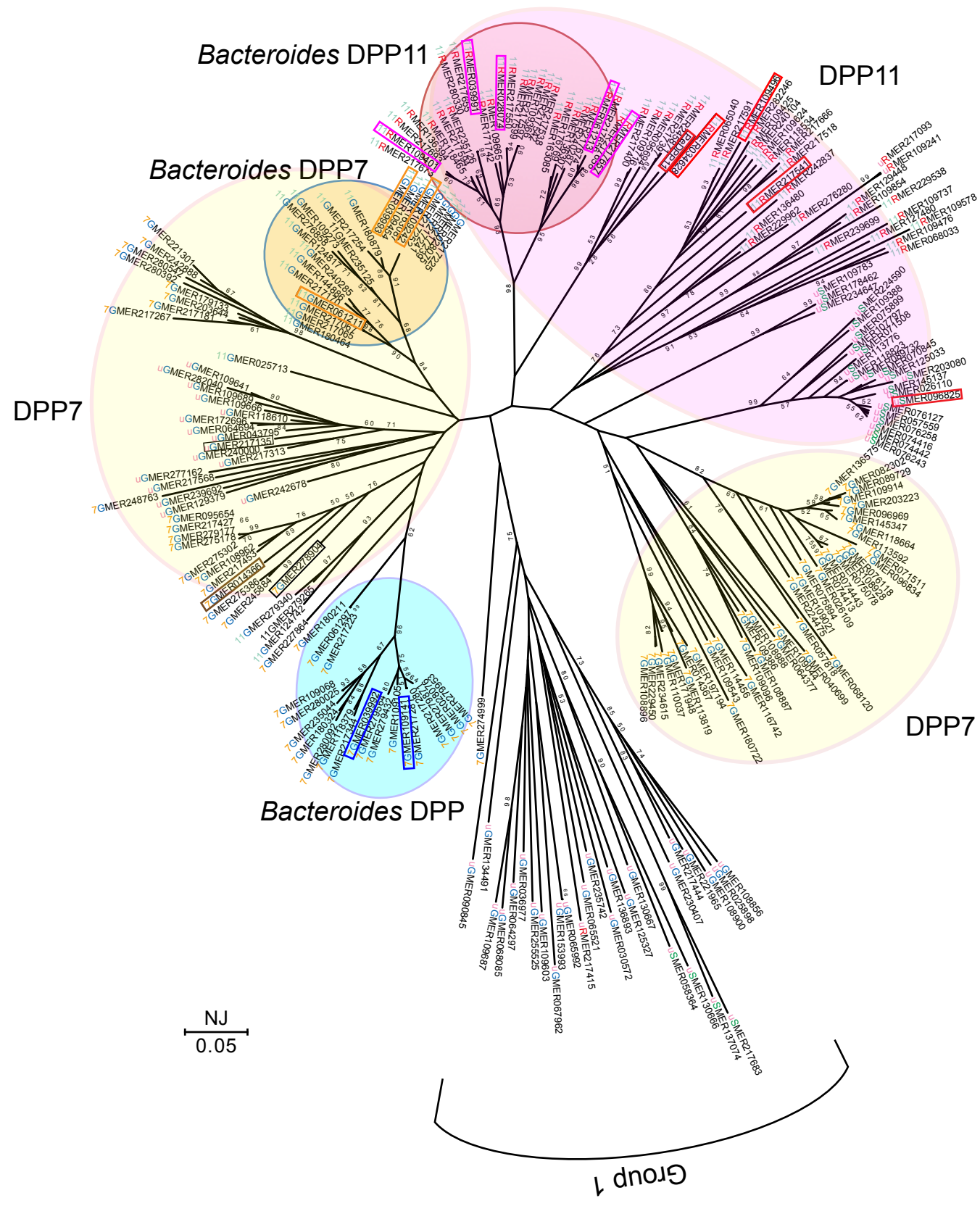

Fig. 7. Phylogenic tree of S46 family based on sequences of C-terminal conserved region.

A phylogenic tree was constructed using the NJ method [25] with ClustalX software [26] based on the C-terminal conserved region ( $\mathrm{Ser}^{571}-\mathrm{Leu}^{700}$ of PgDPP11). Basic information for DPPs is included in the names, as follows: first 7 (DPP7), 11 (DPP11), or $u$ (unassigned) in the MEROPS database (Release 9.6), followed by $\mathrm{G}\left(\mathrm{Gly}{ }^{673}\right), \mathrm{R}\left(\mathrm{Arg}^{673}\right)$, or S $\left(\mathrm{Ser}^{673}\right)$, then finally the MEROPS codes. For example, PgDPP11 with $\mathrm{Arg}^{673}$ is written as 11RMER034628. DPPs 
expressed in the present study are boxed in brown (PgDPP7), orange (Bacteroides DPP7), red (DPP11), pink (Bacteroides-type DPP11), and blue (third group). P. endodontalis (7GMER27890) and C. gingivalis (uGMER218135) DPP7, boxed in black, were previously characterized [20]. DPP7 (light yellow), DPP11 (light pink), Bacteroides DPP as the third group of S46 members (blue), Bacteroides DPP7 (cream), and Bacteroides-type DPP11 (red) clusters are indicated by circles. 